\title{
Single session of action observation in choice reaction time in healthy children
}

\author{
Isabella Franco Silva Vieira ${ }^{1}$ (D), Eduardo de Moura Neto ${ }^{1}$ (D), Tamise Aguiar Caires ${ }^{1}$ (D), \\ Gabriel Jablonski $^{2}$ (D), Adriano Oliveira Andrade ${ }^{2}$ (D), Gustavo José Luvizutto ${ }^{1}$ (D), \\ Luciane Aparecida Pascucci Sande de Souza ${ }^{1}$ (D) \\ ${ }^{1}$ Universidade Federal do Triângulo Mineiro, Departamento de Fisioterapia Aplicada, Uberaba, \\ MG, Brasil; ${ }^{2}$ Universidade Federal de Uberlândia, Núcleo de Inovação e Avaliação Tecnológica \\ em Saúde, Uberlândia, MG, Brasil.
}

Editor: Angelina Zanesco, Universidade Estadual Paulista “Júlio de Mesquita Filho", Instituto de Biociências, Departamento de Educação Física, Rio Claro, SP, Brasil.

\begin{abstract}
Aim: Learning by action observation (AO) is a fundamental cognitive ability existing from birth either in live or in the video. However, the specific AO training, in live or in the video, can influence decision making and motor planning in healthy children? This study aimed to evaluate if a single session of both practices (live and video) modifies the choice reaction time during reaching tasks in healthy children. Methods: This is a cross-sectional and randomized study with 22 children aged 6 to 8 years. We measured the choice reaction time (CRT) by electromyography at baseline and after both practices. Data were analyzed using Friedman and posthoc Dunn non-parametric tests for each age group individually as well as all ages combined. Kurtosis analysis was performed to assess data variability. Results: Significant decrease in CRT was observed after action observation in the video in 8-year-olds. Also, we observed choice reaction time variability reduction in 8-year-olds after both practices compared to that at baseline. Conclusion: A decrease in CRT was observed after the single session of action observation in the video in 8-year-olds. Additionally, there was a reduced variability in CRT after performing both practices in the same age group.
\end{abstract}

Keywords: action observation, learning, children, reaction time, cognitive function.

\section{Introduction}

New competencies may be acquired through learning by observation ${ }^{1}$. An individual transforms the observation into action like the motor strategies to be applied ${ }^{2}$. This process facilitates motor learning, thereby ensuring the acquisition of the main spatial and temporal features of the $\operatorname{task}^{3,4}$.

Learning by observation is a fundamental cognitive ability existing from birth ${ }^{5,6}$. The term action observation (AO) has also been associated with a recent therapy that has already been shown to be effective in improving motor and cognitive function ${ }^{7}$. This is a relevant issue in the field of neurorehabilitation ${ }^{8}$. The AO can be performed live (AOL), wherein an individual watches another individual performing a motor action; or in the video (AOV), wherein the observation is made via a digital interface? The AO of motor actions can activate the corresponding motor representations in the brain due to the mechanism of mirror neurons and improve motor and cognitive respon$\operatorname{ses}^{10}$.

Among the AO applications found in literature, it is noted that it can be used in school-aged children ${ }^{11,12}$. AO requires cognitive function, like attentiveness and planning ${ }^{13,14}$. In this way, the evaluation of choice reaction time (CRT) can measure decision making and motor planning ${ }^{15}$. This competence can be evaluated, especially in the behaviour transitional age, i.e. between 6 and 8 years when the instability gives rise to stability ${ }^{15,16}$ and their CRT tends to decrease. However, certain questions such as can AO interfere with the RT values in this age group and is there a difference between AOV and AOL approaches are yet to be answered.

Therefore, this study aimed to evaluate if a single session of AO in live and video modifies the CRT during reaching tasks in children between 6 and 8 years. This study hypothesized that both AOV and AOL would contribute to reducing the CRT in children of 6 to 8 years.

\section{Methods}

\section{Study design, setting, and participants}

This is an experimental and randomized study with 22 (11 boys and 11 girls) children aged 6 to 8 years of both sexes who are regularly enrolled at the school. This study 
was approved by the Human Research Ethics Committee of the Federal University of Triângulo Mineiro (CAAE: 17240819.2.0000.5154), and the evaluation took place after parental consent of the children. The experiments were performed at the Laboratory of Biomechanics and Motor Control of the Federal University of Triângulo Mineiro.

\section{Eligibility criteria}

Inclusion criteria were: absence of motor, neurological or cognitive impairment, age 6 to 8 years old, Standard Score $\geq 8$ on The Movement Assessment Battery for Children (MABC-2), and not on any prescription medications. We excluded children who did not conclude the tests at any moment.

\section{Variables}

a) Independent: Type of action observation (video and live).

b) Dependent (outcomes): choice reaction time

\section{Procedures}

The procedures are shown below in Figure 1. It is important to emphasize that prior to the training, all children could play and draw for 5 minutes so that they could adapt to the laboratory environment.

\section{Data sources/measurement}

Demographic data

Age, gender, parents' income, evaluation of video vs live watching preference, and technology interaction time were collected by an interview.

\section{CRT evaluation}

The individuals were seated in a chair with adjustable height, with their hips, knees, and ankles at $90^{\circ}$ of flexion, their shoulders between $10^{\circ}$ and $15^{\circ}$ of flexion, their elbows between $75^{\circ}$ and $90^{\circ}$ of flexion, and their forearms pronated. To avoid compensatory movements, the trunk was stabilized at the $7^{\text {th }}$ thoracic vertebra using a chest brace. A monitor was placed in front of the individual at a distance-dependent on the upper limb length, which was measured from the acromion to the distal phalanx of the index finger, using a measuring tape. The seat height was adjusted to $100 \%$ of the lower limb length, which was measured from the lateral knee joint to the floor in a straight line with the subject standing barefoot. The centre of the monitor height was adjusted to $75 \%$ of the subject's shoulder height, which was defined as the distance from the shoulder marker to the floor with the subject sitting in the standardized position. The individual had to reach into the ipsilateral or contralateral spaces, 5 times, in response to the visual stimulus, as quickly and accurately as possible, and return to the anatomic initial position at the end. The visual stimulus, represented by a white circle, lasted 5 seconds (in 15 seconds) and could appear at five, six, seven, eight, or nine seconds in a randomized sequence. It was used only two circles for CRT evaluation (Figure 2).

After the CRT evaluation, the children performed the single session of action observation according to the initial randomization: video or live. After the session, the CRT evaluation was performed again.

The electromyographic signal of the anterior deltoid was used to determine the onset of the muscle response analyzed. The EMG signals were recorded using a Delsys Trigno $\mathrm{TM} \circledast$ wireless telemetry sensor at $2,000 \mathrm{~Hz}$, and they were recorded according to the SENIAM protocol

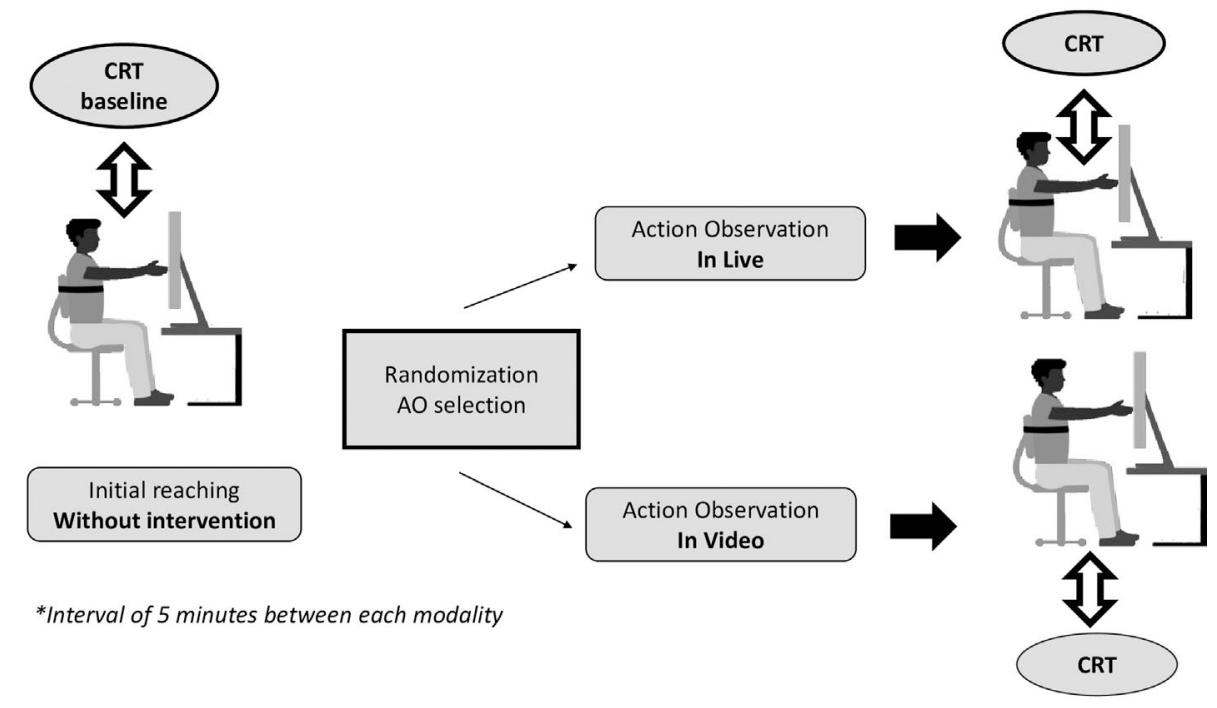

Figure 1 - Study design; Legends: $\mathrm{CRT}=$ choice reaction time; $\mathrm{AO}=$ action observation. 


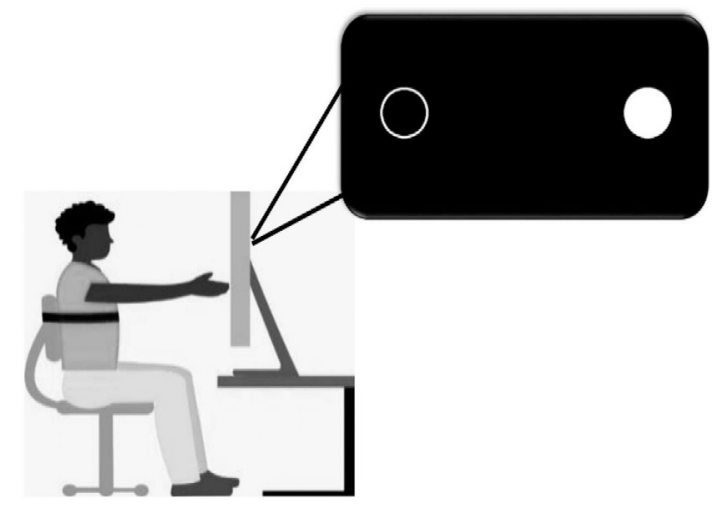

Figure 2 - Choice reaction time analysis.

(Surface Electromyography for the Non-invasive Assessment of Muscles) ${ }^{17}$. A photodiode was used to synchronize the EMG signal with the visual stimulus.

The CRT in milliseconds was calculated by the difference between the photodiode signal and EMG activity in three reaching tasks:

(1) before action observation (baseline)

(2) after action observation (in video or live)

(3) after action observation (in video or live)

\section{Action observation protocol}

The AO protocol was implemented live and in the video.

a) Action observation in live (AOL): the children observed the researcher perform reaching movements into the ipsilateral or contralateral spaces in response to the visual stimulus for 1 minute and 30 seconds. The children were positioned behind the researcher.

b) Action observation in the video (AOV): the children observed the same action described under point a) but using the video interface. The video was placed in the same visual perspective as watching live action for 1 minute and 30 seconds.

All children performed both practices (AOV and AOL), randomized by a computer generator. The same setting was applied in both tasks.

\section{Statistical analysis}

The Kolmogorov and Smirnov tests were performed, and normality was not observed. Choice RT was considered in the three situations: baseline, AOV, and AOL. Then, the Friedman and post-hoc Dunn non-parametric tests were performed for each age $(6,7,8$ years old) and all ages combined, considering $\mathrm{p}<0.05$. In addition, kurtosis $(\mathrm{k})$ was also calculated for data variability analysis. Data were analyzed with IBM SPSS Statistics® Version 21 and Prism 7 software.

\section{Results}

The sample consisted of twenty-two children (11 boys and 11 girls), with an average age of $7.04 \pm$ 0.84 years old $(31.7 \%$ for 6 years; $31.7 \%$ for 7 years; and $36.6 \%$ for 8 years); average weight of $25.5 \pm 6.2 \mathrm{~kg}$; and the average height of $1.25 \pm 0.07 \mathrm{~m}$. Overall, fifteen children preferred watching the action observation in live $(68.2 \%)$, while seven preferred videos $(31.8 \%)$. The parents of the children selected for this study had an average income above 6 minimum salaries, varying from the middle-income to upper-income group, according to IBGE (2019). The time children spent on technology interaction in a day varied according to their ages, with 20 to $30 \mathrm{~min}$ utes in children of 6 years, 50 to 60 minutes in children of 7 years, and 2 to 3 hours in children of 8 years. The technology interaction included watching videos on YouTube and playing games.

After analysis without separating by age, no statistically significant difference in choice RT was observed between baseline and AOL $(p=0.07)$, baseline and AOV $(p=0.07)$, and AOL and AOV $(p>0.99)$ (Figure 3A). In the analysis of each age separately, there was no statistically significant difference in any of the comparisons for the 6-year-old children (Baseline $\mathrm{x}$ AOL, $\mathrm{p}=0.63$; Baseline $\mathrm{x} \mathrm{AOV}, \mathrm{p}=0.24$; AOL $\mathrm{x} \mathrm{AOV}, \mathrm{p}>0.99$ ) (Figure 3B) and for the 7-year-old children (Baseline $\mathrm{x}$ AOL, $\mathrm{p}=0.95$; Baseline $x$ AOV, p > 0.99, AOL $x$ AOV, p > 0.99) (Figure 3C). In the 8-year-old children, a reduction in the choice RT was observed after AOV compared to that at baseline $(p=0.03)$. There was no statistical significance in the other comparisons: Baseline $\mathrm{x}$ AOL $(\mathrm{p}=0.13)$ and AOL x AOV ( $p>0.99)$ (Figure 3D).

Due to the high variability of the choice RT values in this sample, the calculation of the kurtosis $(\mathrm{k})$ was also applied. The values of $\mathrm{k}$ are shown in Table 1 . We can observe that there is no change in the variability of the data considering all ages together and observing children aged 6 and 7 years separately. However, in the 8-year-old children, it is noted that both AOV and AOL contributed to reducing the $\mathrm{k}$ variability compared to that at baseline.

\section{Discussion}

Based on our results, we can say that our initial hypothesis that the choice RT could decrease after AOV and AOL interventions was partially confirmed because there was a significant reduction in the choice RT after the AOV in 8-year-old children. Additionally, in this specific age group, there was a reduction in the variability of the choice RT values after AOV and AOL practices.

Our findings highlighted the impact of AOV on choice RT of anterior deltoid in children aged 8 years. Hay et al. ${ }^{18}$ observed that the RT in reaching during different types of motor activities decreases in children from 6 to 

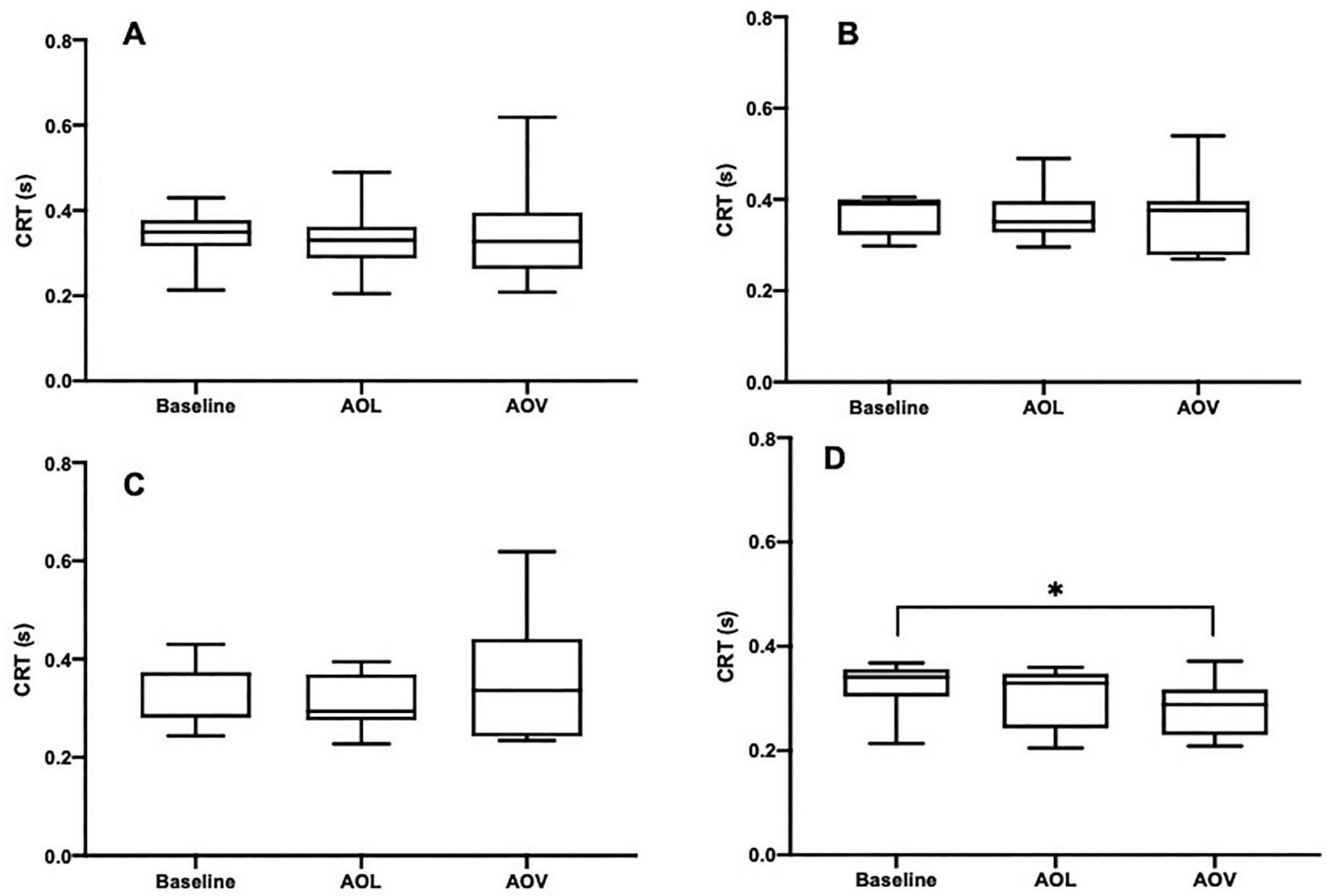

Figure 3 - Choice reaction time before and after AO intervention (in live and in the video); A: all children; B: 6 years old; C: 7 years old; D: 8 years old.

Table 1 - Kurtoses variability before and after the action observation (live and video).

\begin{tabular}{lcccc}
\hline Kurtoses & All ages & 6 years & 7 years & 8 years \\
\hline Baseline & 0.588 & -1.359 & 0.061 & 3.794 \\
After AOL & 0.944 & 2.483 & -0.634 & -0.510 \\
After AOV & 1.589 & 2.010 & 0.790 & -0.533
\end{tabular}

Legends: AOL: action observation in live; AOV: action observation in video.

10 years old with advancing age, with significant changes being observed up to about 8 to 9 years old. In our study, the two types of AO practice had no impact on RT and its variability in children aged 6 and 7 years, probably due to the behavioural transition period. In the study of Gribble and Mattar ${ }^{18}$, with a sample of adults, the authors observed better motor performance after $\mathrm{AO}$ in the video compared to that after non-AO practice. Favilla ${ }^{19}$ observed that the precision and accuracy of motor and cognitive tasks also temporarily decreased at 7 years old, followed by an increase at 8 years. At 9 years old, both precision and RT reached levels close to those of adults.

In addition, the lower variability after the two $\mathrm{AO}$ practices in these 8-year-old children may indicate a more organized RT behaviour in the reaching task. In other words, children who had highly variable values in the baseline showed greater uniformity and symmetry of choice RT values after both practices. In this sense, considering that 8-year-olds are in the transition from motor behaviour to adulthood pattern, we can relate these findings to better motor performance. Frossberg and Nashner ${ }^{20}$ also found a higher RT variability in the children compared to the adults. These authors attribute these findings to the neurophysiological effect that the nerve conduction velocity of children is lower20. Such variability may be linked to developmental immaturity ${ }^{21}$.

Like the behaviour of choice RT in this study, the declarative spatial competence is not yet fully developed in children younger than about 7 years of age ${ }^{22}$. Children from 8 to 10 years of age displayed increased neural activity in cerebral areas associated with visuospatial processing ${ }^{23}$, which could contribute to the reduction of choice RT in 8-year-old children.

A question that comes to mind is: is the greater knowledge of digital technologies and time spent using them the reason for the shortest reaction times in these children?

In the last decade, the use of new technologies (interactive and digital media) has increased exponentially ${ }^{24}$. The majority of parents still reported that their children watched TV for 2 or more hours per day, with $54 \%$ of children from middle- and high-income families making frequent use of educational content, while the same 
content was used by only $28 \%$ of children from lowincome families ${ }^{24,25}$. Although there are many negative points among the good aspects of digital media for children, Chassiakos et al. ${ }^{24}$ showed that the use of digital and social media can improve developmental outcomes in children. However, we must determine what content, interactions, and amount of time spent are appropriate.

One of the main limitations of this study was the small sample size. In future studies with the same approach, we suggest testing another agonist of reaching the task. And also, as stated by Lagravinese ${ }^{26}$ AO movement speed has an impact on the outcome, and we did not control such variable. However, this research has produced potential contributions, allowing comparisons of CRT in healthy children undergoing AO training. In addition, the OA (video or live approach) can be a strategy for several neurological conditions that reduce CRT and cognitive function.

\section{Conclusion}

A decrease in choice RT was observed after a single session of $\mathrm{AO}$ in the video in 8-year-old children. In addition, there was a reduction in variability in choice RT after $\mathrm{AOV}$ and AOL in 8-year-old children. In the other age groups, there were no changes in the choice RT after AO practice.

\section{Acknowledgments}

Would like to thanks the Biomechanics and Motor control laboratory to support our research

\section{References}

1. Bandura A. Self-efficacy: toward a unifying theory of behavioral change. Psychol Rev. 1977;84(2):191-215. doi: 10.1037//0033-295x.84.2.191.

2. Meltzoff AN, Decety J. What imitation tells us about social cognition: a rapprochement between developmental psychology and cognitive neuroscience. Philos Trans R Soc Lond B Biol Sci. 2003;358(1431):491-500. doi: 10.1098/ rstb.2002.1261. PMID: 12689375.

3. Buchanan JJ, Dean NJ. Specificity in practice benefits learning in novice models and variability in demonstration benefits observational practice. Psychol Res. 2010;74 (3):313-26. doi: 10.1007/s00426-009-0254-y.

4. Rohbanfard H, Proteau L. Learning through observation: a combination of expert and novice models favors learning. Exp Brain Res. 2011;215(3-4):183-97. doi: 10.1007/ s00221-011-2882-x.

5. Meltzoff AN, Kuhl PK, Movellan J, Sejnowski TJ. Foundations for a new science of learning. Science. 2009;325 (5938):284-8. doi: 10.1126/science.1175626.

6. Nadel J. Imitation and imitation recognition: functional use in preverbal infants and nonverbal children with autism. The Imitative Mind: Development, Evolution, and Brain Bases.
In: Meltzoff A, Prinz W (Eds.). The Imitative Mind Cambridge: Cambridge University Press, 2002; pp. 42-62.

7. Sarasso E, Gemma M, Agosta F, Filippi M, Gatti R. Action observation training to improve motor function recovery: a systematic review. Arch Physiother. 2015;5:14. doi: 10.1186/s40945-015-0013-x.

8. Celnik P, Stefan K, Hummel F, Duque J, Classen J, Cohen LG. Encoding a motor memory in the older adult by action observation. Neuroimage. 2006;29(2):677-84. doi: 10.1016/ j.neuroimage.2005.07.039.

9. Kim DH, An DH, Yoo WG. Effects of live and video form action observation training on upper limb function in children with hemiparetic cerebral palsy. Technol Health Care. 2018;26(3):437-443. doi: 10.3233/THC-181220.

10. Fabbri-Destro M, Rizzolatti G. Mirror neurons and mirror systems in monkeys and humans. Physiology (Bethesda). 2008;23:171-9. doi: 10.1152/physiol.00004.2008.

11. Buccino G. Action observation treatment: a novel tool in neurorehabilitation. Philos Trans R Soc Lond B Biol Sci. 2014;369(1644):20130185. doi: 10.1098/rstb.2013.0185.

12. Buccino G, Arisi D, Gough P, Aprile D, Ferri C, Serotti L, Tiberti A, Fazzi E. Improving upper limb motor functions through action observation treatment: a pilot study in children with cerebral palsy. Dev Med Child Neurol. 2012;54 (9):822-8. doi: 10.1111/j.1469-8749.2012.04334.x.

13. Foti F, Menghini D, Orlandi E, Rufini C, Crinò A, Spera S, Vicari S, Petrosini L, Mandolesi L. Learning by observation and learning by doing in Prader-Willi syndrome. J Neurodev Disord. 2015;7(1):6. doi: 10.1186/s11689-015-9102-0.

14. Torriero S, Oliveri M, Koch G, Caltagirone C, Petrosini L. What and how of observational learning. J Cogn Neurosci. 2007 Oct;19(10):1656-63. doi: 10.1162/ jocn.2007.19.10.1656.

15. Haywood KM, Getchell N. Desenvolvimento Motor ao

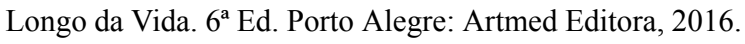

16. Shumway-cook A, Woollacott MH. Alcance, preensão e manipulação normais. Controle motor: teoria e aplicações práticas, 2010; p.449-473.

17. Hermens HJ, Freriks B, Disselhorst-Klug C, Rau G. Development of recommendations for SEMG sensors and sensor placement procedures. J Electromyogr Kinesiol. 2000 Oct;10(5):361-74. doi: 10.1016/s1050-6411(00)00027-4.

18. Hay L, Bard C, Fleury M. Visuo-manual coordination from 6 to 10: Specification, control, and evaluation of direction and amplitude parameters of movement. Motor development in children: Aspects of coordination and control. 1986; 319-338. doi: 10.1007/978-94-009-4460-2_18

19. Mattar AA, Gribble PL. Motor learning by observing. Neuron. 2005;46(1):153-60. doi: 10.1016/j.neuron.2005.02.009.

20. Favilla M. Reaching movements in children: accuracy and reaction time development. Exp Brain Res. 2006;169 (1):122-5. doi: 10.1007/s00221-005-0291-8.

21. Forssberg H, Nashner LM. Ontogenetic development of postural control in man: adaptation to altered support and visual conditions during stance. J Neurosci. 1982;2(5):54552. doi: 10.1523/JNEUROSCI.02-05-00545.1982.

22. Barrett PT, Daum I, Eysenck HJ. Sensory nerve conduction and intelligence: a methodological study. J Psychophysiol. 1990;4:1-13. 
23. Klingberg T. Development of a superior frontal-intraparietal network for visuospatial working memory. Neuropsychologia. 2006;44(11):2171-7. doi: 10.1016/j.neuropsychologia.2005.11.019.

24. Murias K, Slone E, Tariq S, Iaria G. Development of spatial orientation skills: an fMRI study. Brain Imaging Behav. 2019 Dec;13(6):1590-1601. doi: 10.1007/s11682-018-00285.

25. Reid Chassiakos YL, Radesky J, Christakis D, Moreno MA, Cross C; COUNCIL ON COMMUNICATIONS AND MEDIA. Children and Adolescents and Digital Media. Pediatrics. 2016 Nov;138(5):e20162593. doi: 10.1542/ peds.2016-2593. PMID: 27940795.

26. Loprinzi PD, Davis RE. Secular trends in parent-reported television viewing among children in the United States, 2001-2012. Child Care Health Dev. 2016;42(2):288-91. doi: 10.1111/cch.12304.

27. Lagravinese G, Bisio A, Ruggeri P, Bove M, Avanzino L. Learning by observing: the effect of multiple sessions of action-observation training on the spontaneous movement tempo and motor resonance. Neuropsychologia. 2017;96:89-95. doi: 10.1016/j.neuropsychologia.2016.09.022.

\section{Corresponding Author}

Gustavo José Luvizutto. Departamento de Fisioterapia Aplicada. Rua Vigário Carlos 100, - Sala 319, $3^{\circ}$ andar, 38025-350 Bairro Abadia; Uberaba, MG, Brasil.

E-mail: gluvizutto@gmail.com.

Manuscript received on December 13, 2020

Manuscript accepted on April 26, 2021

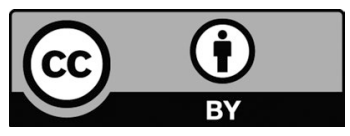

Motriz. The Journal of Physical Education. UNESP. Rio Claro, SP, Brazil - eISSN: 1980-6574 - under a license Creative Commons - Version 4.0 